\title{
Discrete-Time Predator-Prey Model with Bifurcations and Chaos
}

\author{
K. S. Al-Basyouni ${ }^{1}$ and A. Q. Khan $\mathbb{D}^{2}$ \\ ${ }^{1}$ Department of Mathematics, King Abdulaziz University, P.O Box 80203, Jeddah 21589, Saudi Arabia \\ ${ }^{2}$ Department of Mathematics, University of Azad Jammu and Kashmir, Muzaffarabad 13100, Pakistan
}

Correspondence should be addressed to A. Q. Khan; abdulqadeerkhan1@gmail.com

Received 13 September 2020; Revised 2 October 2020; Accepted 11 October 2020; Published 16 November 2020

Academic Editor: Cemil Tunç

Copyright (c) 2020 K. S. Al-Basyouni and A. Q. Khan. This is an open access article distributed under the Creative Commons Attribution License, which permits unrestricted use, distribution, and reproduction in any medium, provided the original work is properly cited.

\begin{abstract}
In this paper, local dynamics, bifurcations and chaos control in a discrete-time predator-prey model have been explored in $\mathbb{R}_{+}^{2}$. It is proved that the model has a trivial fixed point for all parametric values and the unique positive fixed point under definite parametric conditions. By the existing linear stability theory, we studied the topological classifications at fixed points. It is explored that at trivial fixed point model does not undergo the flip bifurcation, but flip bifurcation occurs at the unique positive fixed point, and no other bifurcations occur at this point. Numerical simulations are performed not only to demonstrate obtained theoretical results but also to tell the complex behaviors in orbits of period-4, period-6, period-8, period-12, period-17, and period-18. We have computed the Maximum Lyapunov exponents as well as fractal dimension numerically to demonstrate the appearance of chaotic behaviors in the considered model. Further feedback control method is employed to stabilize chaos existing in the model. Finally, existence of periodic points at fixed points for the model is also explored.
\end{abstract}

\section{Introduction}

In the mid-1920s, an Italian Biologist U. D'Ancona studied the population variations in different species of fish that were interacting with one another. In his investigation, he found some percentage data of total catch of different fish species during the World War I which was brought into various Mediterranean ports. In precise, the data determine the percentage (\%) of total catch of selachians, which are not adorable as food fish, and during the years 1914-1923, the percentage data for the port of Fiume, Italy, is given in Table 1.

D'Ancona was surprised by a very large increase in the percentage of selachians during the World War I. He reasoned the increase in the percentage of selachians was because of decline in fishing during this period. But how does the intensity of fishing affect the fish populations? The answer was obviously the struggle for existence between competing species, which was of great concern to D'Ancona and also to the fishing industry. Naturally, selachians depend on food fish for their survival as selachians are predators and food fish are prey. D'Ancona thought that this accounted for the large increase in number of selachians during the war period. Since the level of fishing was less, there were more food fish available to selachians, which therefore increased rapidly. D'Ancona just shows the increase in number of selachians when fishing's level is reduced. D'Ancona did not explain why a declining level of fishing is more helpful to predators than to their prey. Later, paying all possible biological clarifications to this phenomenon, D'Ancona twisted to his coworker, the well-known mathematician Vito Volterra. With hope, Volterra would come up with a mathematical model of the growth of the selachians and food fish, their prey, and his model would provide the answer to D'Ancona's question. Volterra started his analysis on this problem by separating all the fish into the prey population $x(t)$ and the predator population $y(t)$. Then, he reasoned that the food fish do not compete very fast among themselves for their food supply since this is very plentiful, and the density of fish population is not very much. Hence, in the absence of predators, their prey would grow according to the Malthusian law of population growth: $(\mathrm{d} x / \mathrm{d} t)=r x$ for positive constant $r$. Next, Volterra reasoned the number of contacts per unit time between selachians (predators) and 
Table 1: Percentage data for the port of Fiume, Italy, during the years 1914-1923.

\begin{tabular}{lc}
\hline Number of years & \% values \\
\hline 1914 & 11.9 \\
1915 & 21.4 \\
1916 & 22.1 \\
1917 & 21.2 \\
1918 & 36.4 \\
1919 & 27.3 \\
1920 & 16.0 \\
1921 & 15.9 \\
1922 & 14.8 \\
1923 & 10.7 \\
\hline
\end{tabular}

food fish (prey) is $b x y$ for positive constant $b$. Therefore, $(\mathrm{d} x / \mathrm{d} t)=r x-b x y$, and so Volterra reasoned that predators have a characteristic pace of lessening $-\mathrm{d} y$ relative to their present number and that they likewise increment at a rate $c x y$ relative to their present number $y$ and their nourishment supply $x$, and hence, one has [1-5]

$$
\begin{aligned}
& \frac{\mathrm{d} x}{\mathrm{~d} t}=r x-b x y, \\
& \frac{\mathrm{d} y}{\mathrm{~d} t}=c x y-d y .
\end{aligned}
$$

Now include the effects of fishing in (1). It is observed that fishing decreases the population of food fish at a rate $\varepsilon x(t)$ and decreases the population of selachians at a rate $\varepsilon y(t)$, and the parameter $\epsilon$ reflects the intensity of fishing. So, by the effect of fishing, the continuous-time model which is depicted in (1) becomes as follows [1]:

$$
\begin{aligned}
& \frac{\mathrm{d} x}{\mathrm{~d} t}=(r-\varepsilon) x-b x y, \\
& \frac{\mathrm{d} y}{\mathrm{~d} t}=c x y-(d+\varepsilon) y .
\end{aligned}
$$

It is important here to note that generally many biological models are directed by continuous as well as discretetime systems, and in recent years, many authors gave great contribution towards discrete models [6-15]. The reasons are that, for nonoverlapping generation, discrete models are much convincing than continuous models, and moreover, these models provide more effective computational results for numerical simulations [6,16-21]. Due to scientific computation, in the present study, our aim is to explore the qualitative behavior of the discrete-time model corresponding to (2). It is predicted that the discrete model is dynamically consistent with the continuous-time model. It is also mentioned in [15] that if population has a nonoverlapping generation, as stated above, it is essential to write a discrete system that corresponds to model (2). So, applying the forward Euler scheme, (2) becomes as follows:

$$
\begin{aligned}
& \frac{x_{t+1}-x_{t}}{h}=(r-\varepsilon) x_{t}-b x_{t} y_{t}, \\
& \frac{y_{t+1}-y_{t}}{h}=c x_{t} y_{t}-(d+\varepsilon) y_{t} .
\end{aligned}
$$

After some manipulations, from (3), one gets

$$
\begin{aligned}
& x_{t+1}=(1+h(r-\varepsilon)) x_{t}-b h x_{t} y_{t}, \\
& y_{t+1}=(1-h(d+\varepsilon)) y_{t}+c h x_{t} y_{t} .
\end{aligned}
$$

This paper is organized as follows: Section 2 is about the topological classifications at fixed points of the model (4), whereas existence of possible bifurcations at respective fixed points is given in Section 3. The comprehensive bifurcation analysis at a positive fixed point is investigated in Section 4. In Section 5, some simulations are performed to demonstrate obtained theoretical results, and this is also about the study of fractal demission that characterized the strange attractors. In Section 6, we investigated the chaos control by the feedback control method, whereas existence of periodic points is studied in Section 7. The conclusion of the paper is given in Section 8.

\section{Topological Classifications at Fixed Points}

We will study the topological classifications at fixed points of the model (4) in this section. In order to determine fixed points, one need to solve the following system, where $(\hat{x}, \hat{y})$ is the fixed point of (4):

$$
\begin{aligned}
& \widehat{x}=(1+h(r-\varepsilon)) \hat{x}-b h \hat{x} \hat{y}, \\
& \hat{y}=(1-h(d+\varepsilon)) \hat{y}+\operatorname{ch} \hat{x} \hat{y} .
\end{aligned}
$$

It is noted that $(\hat{x}, \hat{y})=(0,0)$, satisfying (5) obviously, and so $E_{\text {trival }}=(0,0)$ is a trivial fixed point of $(4)$. For a positive fixed point, system (5) reduces into the following form:

$$
\begin{aligned}
h(r-\varepsilon)-b h \hat{y} & =0, \\
-h(d+\varepsilon)+c h \hat{x} & =0 .
\end{aligned}
$$

From (6), one gets $x=((d+\varepsilon) / c)$ and $y=((r-\varepsilon) / b)$. Therefore, (4) has the unique positive fixed point: $E_{\text {positive }}=$ $(((d+\varepsilon) / c),((r-\varepsilon) / b))$ if $r>\epsilon$.

Hereafter, we will study the topological classifications at $E_{\text {trival }}$ and $E_{\text {positive }}$ of the model (4) by method of linearization. So, the Jacobian matrix $\left.\Omega\right|_{E_{(\widehat{x}, y)}}$ evaluated at $E_{(\widehat{x}, \widehat{y})}$ becomes as follows:

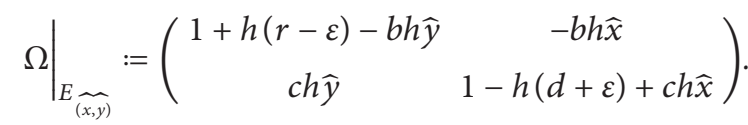

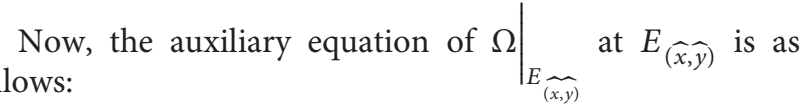

$$
\zeta^{2}-\mathscr{T}\left(E_{(\widehat{x}, \widehat{y})}\right) \zeta+\mathscr{D}\left(E_{(\widehat{x}, \hat{y})}\right)=0,
$$

where

$$
\begin{aligned}
& \mathscr{T}\left(E_{(\widehat{x}, \hat{y})}\right)=2+h r-2 h \varepsilon-b h \hat{y}-h d+c h \hat{x}, \\
& \mathscr{D}\left(E_{(\widehat{x}, \hat{y})}\right)=(1+h(r-\varepsilon)-b h \hat{y})(1-h(d+\varepsilon)+c h \hat{x})+b \operatorname{ch}^{2} \hat{x} \hat{y} .
\end{aligned}
$$


Now, in the rest of the section, we will give topological classification at equilibria: $E_{\text {trival }}$ and $E_{\text {positive }}$ as follows:

2.1. Topological Classifications at $E_{\text {trival }}$. It is noted that, at $E_{\text {trival }}$, (7) takes the following form:

$$
\left.\Omega\right|_{E_{\text {trival }}}:=\left(\begin{array}{cc}
1+h(r-\varepsilon) & 0 \\
0 & 1-h(d+\varepsilon)
\end{array}\right),
$$

whose characteristic roots are $\zeta_{1}=1+h(r-\varepsilon)$ and $\zeta_{2}=1-h(d+\varepsilon)$. So based on stability theory, one can conclude the topological classifications at $E_{\text {trival }}$ as the following result.

Proposition 1. For $E_{\text {trival }}$, the following classifications hold:

(I) If

$$
0<h<\min \left\{\frac{2}{\varepsilon-r}, \frac{2}{d+\varepsilon}\right\} \text {, where } \varepsilon>r \text {, }
$$

then $E_{\text {trival }}$ is a sink.

(II) If

$$
h>\max \left\{\frac{2}{\varepsilon-r}, \frac{2}{d+\varepsilon}\right\}, \quad \text { where } \varepsilon>r,
$$

then $E_{\text {trival }}$ is a source.

(III) If

$$
\frac{2}{\varepsilon-r}<h<\frac{2}{d+\varepsilon}
$$

then $E_{\text {trival }}$ is a saddle.

(IV) If

$$
h=\frac{2}{\varepsilon-r}
$$

or

$$
h=\frac{2}{d+\varepsilon},
$$

then $E_{\text {trival }}$ is nonhyperbolic.

2.2. Topological Classifications at $E_{\text {positive }}$ Now, we will give topological classifications at $E_{\text {positive }}$ for the considered system. After some manipulations, at $E_{\text {positive }}$ (7) takes the following form:

$$
\left.\Omega\right|_{E_{\text {positive }}}:=\left(\begin{array}{cc}
1 & -\frac{b h}{c}(d+\varepsilon) \\
\frac{c h}{b}(r-\varepsilon) & 1
\end{array}\right) .
$$

Furthermore, at $E_{\text {positive }}$, (8) becomes

$$
\zeta^{2}-\mathscr{T}\left(E_{\text {positive }}\right) \zeta+\mathscr{D}\left(E_{\text {positive }}\right)=0,
$$

where

$$
\begin{aligned}
& \mathscr{T}\left(E_{\text {positive }}\right)=2, \\
& \mathscr{D}\left(E_{\text {positive }}\right)=1+h^{2}(r-\varepsilon)(d+\varepsilon) .
\end{aligned}
$$

Finally, from (17) one gets

$$
\zeta_{1,2}=\frac{2 \pm \sqrt{\mathrm{Disc}}}{2},
$$

where

$$
\begin{aligned}
\text { Disc } & :=\left(\mathscr{T}\left(E_{\text {positive }}\right)\right)^{2}-4 \mathscr{D}\left(E_{\text {positive }}\right) \\
& =4 h^{2}(\varepsilon-r)(d+\varepsilon) .
\end{aligned}
$$

Hereafter, at equilibrium: $E_{\text {positive }}$, we will summarize the topological classifications by allowing sign of discriminant quantity, i.e., Disc $=4 h^{2}(\varepsilon-r)(d+\varepsilon)<0$ (respectively $\left.\geq 0\right)$, as a following proposition.

Proposition 2. If Disc $=4 h^{2}(\varepsilon-r)(d+\varepsilon)<0$, then for $E_{\text {positive }}$, the following classifications hold:

(I) $E_{\text {positive }}$ is never stable focus.

(II) If

$$
r>\varepsilon
$$

then $E_{\text {positive }}$ is unstable focus.

(III) If

$$
r=\varepsilon
$$

then $E_{\text {positive }}$ is nonhyperbolic.

Proposition 3. If Disc $=4 h^{2}(\varepsilon-r)(d+\varepsilon) \geq 0$, then for $E_{\text {positive }}$, the following classifications hold:

(I) If

$$
r>\frac{\varepsilon h^{2}(d+\varepsilon)-4}{h^{2}(d+\varepsilon)}
$$

with 


$$
d>\frac{4-\varepsilon^{2} h^{2}}{\varepsilon h^{2}}
$$

then $E_{\text {positive }}$ is a stable node.

(II) If

$$
r<\frac{\varepsilon h^{2}(d+\varepsilon)-4}{h^{2}(d+\varepsilon)}
$$

along with (24) holds, then $E_{\text {positive }}$ is an unstable node.

(III) If

$$
r=\frac{\varepsilon h^{2}(d+\varepsilon)-4}{h^{2}(d+\varepsilon)}
$$

then $E_{\text {positive }}$ is nonhyperbolic.

\section{Existence of Possible Bifurcations at Fixed Points: $E_{\text {trival }}$ and $E_{\text {positive }}$}

In view of obtained results in Section 2 regarding topological classifications at equilibria $E_{\text {trival }}$ and $E_{\text {positive }}$, we will study the existence of possible bifurcations in this section, as follows:

(I) Recall that, at $E_{\text {trival }},\left.\Omega\right|_{E_{\text {trival }}}$ has two characteristic roots in which $\left.\quad \zeta_{1}\right|_{(14)}=-1, \quad$ but $\left.\zeta_{2}\right|_{(14)}=1-(2 / \varepsilon-r)(d+\varepsilon) \neq-1$ or 1 . This implies that (4) may undergo the flip bifurcation if $(b, c, d, h, r, \varepsilon)$ locate in the set:

$$
\left.\mathrm{FB}\right|_{E_{\text {trival }}}=\left\{(b, c, d, h, r, \varepsilon), h=\frac{2}{\varepsilon-r}\right\},
$$

or

Again under the nonhyperbolic condition, which is depicted in (15), one can obtain that $\left.\zeta_{1}\right|_{(15)}=1+(2 / d+\varepsilon)(r-\varepsilon) \neq-1$ or $1, \quad$ but $\zeta_{2}{ }_{(15)}=-1$. This implies that model (4) may undergo the flip bifurcation if $(b, c, d, h, r, \varepsilon)$ locate in the set:

$$
\left.\mathrm{FB}\right|_{E_{\text {trival }}}=\left\{(b, c, d, h, r, \varepsilon), h=\frac{2}{d+\varepsilon}\right\} .
$$

(II) Under the hypothesis of Proposition 2 and obtained nonhyperbolic condition, which is depicted in (22), one gets $\left|\zeta_{1,2}\right|_{(22)}=1$. Hence, model (4) may undergo a Neimark-Sacker bifurcation if parameters $(b, c, d, h, r, \varepsilon)$ locate in the set:

$$
\left.\mathrm{HB}\right|_{E_{\text {positive }}}=\{(b, c, d, h, r, \varepsilon), r=\varepsilon\} .
$$

(III) Under the hypothesis of Proposition 3 and obtained nonhyperbolic condition, which is depicted in (26), one gets $\left.\zeta_{1}\right|_{(26)}=-1$, but $\left.\zeta_{2}\right|_{(26)}=3 \neq-1$ or 1 . So model (4) may undergo the flip bifurcation if $(b, c, d, h, r, \varepsilon)$ locate in the set:

$$
\left.\mathrm{FB}\right|_{E_{\text {positive }}}=\left\{(b, c, d, h, r, \varepsilon), r=\frac{\varepsilon h^{2}(d+\varepsilon)-4}{h^{2}(d+\varepsilon)}\right\} .
$$

Note: for case I, it is easy to see that model does not undergo flip bifurcation if $(b, c, d, h, r, \varepsilon) \in \mathrm{FB} \mid E_{\text {trival }}$, and hence, $E_{\text {trival }}$ is degenerated with a higher codimension.

In the subsequent section, we will present comprehensive N-S and flip bifurcations analysis when parameters, respectively, $\left.\quad(b, c, d, h, r, \varepsilon) \in \mathrm{HB}\right|_{E_{\text {positive }}}$ and $(b, c$, $d, h, r, \varepsilon)\left.\in \mathrm{FB}\right|_{E_{\text {positive }}}$.

\section{Comprehensive Bifurcation Analysis at $E_{\text {positive }}$}

4.1. N-S Bifurcation at $E_{\text {positive }}$. In the subsequent section, we will discuss N-S bifurcation of model (4) at $E_{\text {positive }}$ if $\left.(b, c, d, h, r, \varepsilon) \in H B\right|_{E_{\text {positive }}}$, and hence, the result can be stated as the following theorem.

Theorem 1. If $\left.(b, c, d, h, r, \varepsilon) \in H B\right|_{E_{\text {positive }}}$ then at $E_{\text {positive, }}$ model (4) does not undergo N-S bifurcation.

Proof. Since $\left.(b, c, d, h, r, \varepsilon) \in H B\right|_{E_{\text {positive }}}$ and hence if bifurcation parameter $r$ varies in a small neighborhood of $r^{*}$, that is, $r=r^{*}+\tau$, where $\tau \ll 1$, then (4) becomes as follows:

$$
\begin{aligned}
& x_{t+1}=\left(1+h\left(r^{*}+\tau-\varepsilon\right)\right) x_{t}-b h x_{t} y_{t}, \\
& y_{t+1}=(1-h(d+\varepsilon)) y_{t}+c h x_{t} y_{t},
\end{aligned}
$$

with $E_{\text {positive }}(\tau)=\left((d+\varepsilon / c),\left(r^{*}+\tau-\varepsilon / b\right)\right)$ if $r^{*}+\tau>\varepsilon$. The auxiliary equation of $\left.\Omega\right|_{E_{\text {positive }}}(\tau)$ at $E_{\text {positive }}(\tau)$ becomes

$$
\zeta^{2}-\mathscr{T}\left(E_{\text {positive }}(\tau)\right) \zeta+\mathscr{D}\left(E_{\text {positive }}(\tau)\right)=0,
$$

where

$$
\begin{array}{r}
\mathscr{T}\left(E_{\text {positive }}(\tau)\right)=2, \\
\mathscr{D}\left(E_{\text {positive }}(\tau)\right)=1+h^{2}\left(r^{*}+\tau-\varepsilon\right)(d+\varepsilon) .
\end{array}
$$

Finally, from (32), one gets

$$
\zeta_{1,2}(\tau)=1 \pm h \sqrt{\left(r^{*}+\tau-\varepsilon\right)(d+\varepsilon)} \iota .
$$

From (34), we obtain 


$$
\begin{aligned}
\left|\zeta_{1,2}\right| & :=\sqrt{\mathscr{D}\left(E_{\text {positive }}(\tau)\right)}, \\
\left.\frac{d\left|\zeta_{1,2}\right|}{\mathrm{d} \tau}\right|_{\tau=0}: & :=\frac{h^{2}}{2}(d+\varepsilon)>0 .
\end{aligned}
$$

Hereafter by using the following transformation

$$
\begin{aligned}
& \Phi_{t}=x_{t}-\frac{d+\varepsilon}{c} \\
& \Psi_{t}=y_{t}-\frac{r^{*}+\tau-\varepsilon}{b},
\end{aligned}
$$

transform $E_{\text {positive }}(\tau)$ in origin. In view of (36), from (31), one gets

$$
\begin{aligned}
& \Phi_{t+1}=\left(1+h\left(r^{*}+\tau-\varepsilon\right)\right)\left(\Phi_{t}+\frac{d+\varepsilon}{c}\right)-b h\left(\Phi_{t}+\frac{d+\varepsilon}{c}\right)\left(\Psi_{t}+\frac{r^{*}+\tau-\varepsilon}{b}\right)-\frac{d+\varepsilon}{c} \\
& \Psi_{t+1}=(1-h(d+\varepsilon))\left(\Psi_{t}+\frac{r^{*}+\tau-\varepsilon}{b}\right)+\operatorname{ch}\left(\Phi_{t}+\frac{d+\varepsilon}{c}\right)\left(\Psi_{t}+\frac{r^{*}+\tau-\varepsilon}{b}\right)-\frac{r^{*}+\tau-\varepsilon}{b} .
\end{aligned}
$$

Now, we will study normal form of (37) if $\tau=0$. After Taylor series expansion about $\left(\Phi_{t}, \Psi_{t}\right)=(0,0)$, one gets

$$
\begin{gathered}
\Phi_{t+1}=\Phi_{t}-\frac{b h}{c}(d+\varepsilon) \Psi_{t}-b h \Phi_{t} \Psi_{t} \\
\Psi_{t+1}=\frac{c h}{b}(r-\varepsilon) \Phi_{t}+\Psi_{t}+\operatorname{ch} \Phi_{t} \Psi_{t} .
\end{gathered}
$$

In order to transform liner part of (38) into canonical form, we use the following transformation that can be easily constructed by computation:

$$
\left(\begin{array}{c}
\Phi_{t} \\
\Psi_{t}
\end{array}\right)=\left(\begin{array}{cc}
-\frac{b h}{c}(d+\varepsilon) & 0 \\
0 & h \sqrt{(r-\varepsilon)(d+\varepsilon)}
\end{array}\right)\left(\begin{array}{c}
X_{t} \\
Y_{t}
\end{array}\right)
$$

In view of (39) and (38),

$$
\left(\begin{array}{c}
X_{t+1} \\
Y_{t+1}
\end{array}\right)=\left(\begin{array}{cc}
1 & h \sqrt{(r-\varepsilon)(d+\varepsilon)} \\
-h \sqrt{(r-\varepsilon)(d+\varepsilon)} & 1
\end{array}\right)\left(\begin{array}{l}
X_{t} \\
Y_{t}
\end{array}\right)+\left(\begin{array}{l}
F\left(X_{t}, Y_{t}\right) \\
G\left(X_{t}, Y_{t}\right)
\end{array}\right)
$$

where

From (41), one gets

$$
\begin{aligned}
& F\left(X_{t}, Y_{t}\right)=-b h^{2} \sqrt{(r-\varepsilon)(d+\varepsilon)} X_{t} Y_{t} \\
& G\left(X_{t}, Y_{t}\right)=-b h^{2}(d+\varepsilon) X_{t} Y_{t} .
\end{aligned}
$$

$$
\begin{aligned}
& \left.\mathrm{F}_{X_{t} X_{t}}\right|_{E_{\text {trival }}}=\left.\mathrm{F}_{Y_{t} Y_{t}}\right|_{E_{\text {trival }}}=0, \\
& \left.\mathrm{~F}_{X_{t} Y_{t}}\right|_{E_{\text {trival }}}=-b h^{2} \sqrt{(r-\varepsilon)(d+\varepsilon)}, \\
& \left.\mathrm{G}_{X_{t} X_{t}}\right|_{E_{\text {trival }}}=\left.\mathrm{G}_{Y_{t} Y_{t}}\right|_{E_{\text {trival }}}=0 \\
& \left.\mathrm{G}_{X_{t} Y_{t}}\right|_{E_{\text {trival }}}=-b h^{2}(d+\varepsilon), \\
& \left.\mathrm{F}_{X_{t} X_{t} X_{t}}\right|_{E_{\text {trival }}}=\left.\mathrm{F}_{X_{t} X_{t} Y_{t}}\right|_{E_{\text {trival }}}=\left.\mathrm{F}_{X_{t} Y_{t} Y_{t}}\right|_{E_{\text {trival }}}=\left.\mathrm{F}_{Y_{t} Y_{t} Y_{t}}\right|_{E_{\text {trival }}}=0 \text {, } \\
& \left.\mathrm{G}_{X_{t} X_{t} X_{t}}\right|_{E_{\text {trival }}}=\left.\mathrm{G}_{X_{t} X_{t} Y_{t}}\right|_{E_{\text {trival }}}=\left.\mathrm{G}_{X_{t} Y_{t} Y_{t}}\right|_{E_{\text {trival }}}=\left.\mathrm{G}_{Y_{t} Y_{t} Y_{t}}\right|_{E_{\text {trival }}}=0 \text {. }
\end{aligned}
$$

In order to undergo the said bifurcation, the following quantity should be nonzero [22-29]: 


$$
\Gamma=-\Re\left(\frac{(1-2 \bar{\zeta}) \bar{\zeta}^{2}}{1-\zeta} \delta_{11} \delta_{20}\right)-\frac{1}{2}\left\|\delta_{11}\right\|^{2}-\left\|\delta_{02}\right\|^{2}+\mathfrak{R}\left(\bar{\zeta} \delta_{21}\right)
$$

where

$$
\begin{aligned}
& \delta_{02}=\left.\frac{1}{8}\left[\mathrm{~F}_{X_{t} X_{t}}-\mathrm{F}_{Y_{t} Y_{t}}+2 \mathrm{G}_{X_{t} Y_{t}}+\iota\left(\mathrm{G}_{X_{t} X_{t}}-\mathrm{G}_{Y_{t} Y_{t}}+2 \mathrm{~F}_{X_{t} Y_{t}}\right)\right]\right|_{E_{\text {trival }}}, \\
& \delta_{11}=\left.\frac{1}{4}\left[\mathrm{~F}_{X_{t} X_{t}}+\mathrm{F}_{Y_{t} Y_{t}}+\iota\left(\mathrm{G}_{X_{t} X_{t}}+\mathrm{G}_{Y_{t} Y_{t}}\right)\right]\right|_{E_{\text {trival }}}, \\
& \delta_{20}=\left.\frac{1}{8}\left[\mathrm{~F}_{X_{t} X_{t}}-\mathrm{F}_{Y_{t} Y_{t}}+2 \mathrm{G}_{X_{t} Y_{t}}+\iota\left(\mathrm{G}_{X_{t} X_{t}}-\mathrm{G}_{Y_{t} Y_{t}}-2 \mathrm{~F}_{X_{t} Y_{t}}\right)\right]\right|_{E_{\text {trival }}}, \\
& \delta_{21}=\frac{1}{16}\left[\mathrm{~F}_{X_{t} X_{t} X_{t}}+\mathrm{F}_{X_{t} Y_{t} Y_{t}}+\mathrm{G}_{X_{t} X_{t} Y_{t}}+\mathrm{G}_{Y_{t} Y_{t} Y_{t}}+\iota\left(\mathrm{G}_{X_{t} X_{t} X_{t}}+\mathrm{G}_{X_{t} Y_{t} Y_{t}}-\mathrm{F}_{X_{t} X_{t} Y_{t}}-\mathrm{F}_{Y_{t} Y_{t} Y_{t}}\right)\right]_{E_{\text {trival }}} .
\end{aligned}
$$

Utilizing (42) in (44), one gets

$$
\begin{aligned}
& \delta_{02}=-\frac{b h^{2}}{4}(d+\varepsilon+\sqrt{(r-\varepsilon)(d+\varepsilon)} \iota), \\
& \delta_{11}=\delta_{21}=0, \\
& \delta_{20}=\frac{b h^{2}}{4}(-d-\varepsilon+\sqrt{(r-\varepsilon)(d+\varepsilon) \iota}) .
\end{aligned}
$$

Using (45) in (43), one gets $\Omega=-\left(b h^{4} / 16\right)(d+\varepsilon)^{2}<0$. Finally, the model considered undergoing N-S bifurcation requires that $\zeta_{1,2}^{m} \neq 1, m=1,2,3,4$ if $\tau=0$ which corresponds to $\mathscr{T}\left(E_{\text {positive }}(0)\right) \neq-2,0,1,2$. However, $\mathscr{T}\left(E_{\text {positive }}(0)\right)=2$ which contradicts to the fact that $\mathscr{T}\left(E_{\text {positive }}(0)\right) \neq-2,0,1,2$, and hence, eigenvalues $\zeta_{1,2}$ of fixed point $(0,0)$ lay in the interaction of the unit circle with the coordinate axes when $\tau=0$. Therefore, model (4) does not undergo N-S bifurcation.

4.2. Flip Bifurcation at $E_{\text {positive. In the subsequent section, we }}$ will discuss flip bifurcation of model (4) at $E_{\text {positive }}$ if $\left.(b, c, d, h, r, \varepsilon) \in \mathrm{FB}\right|_{E_{\text {positive }}}$, and hence, the result can be stated as the following theorem.

Theorem 2. If $\left.(b, c, d, h, r, \varepsilon) \in F B\right|_{E_{\text {positive }}}$ then at $E_{\text {positive }}$, model (4) undergoes the flip bifurcation if $p$ varies in a small neighborhood of the origin.

Proof. It is noted that if $r$ in a small $n b h d$ of $r^{*}$, i.e, $r=r^{*}+p$, where $p \ll 1$, then model (4) becomes

$$
\begin{aligned}
& x_{t+1}=\left(1+h\left(r^{*}+p-\varepsilon\right)\right) x_{t}-b h x_{t} y_{t} \\
& y_{t+1}=(1-h(d+\varepsilon)) y_{t}+c h x_{t} y_{t} .
\end{aligned}
$$

Now, one can transform $E_{\text {positive }}(p)$ in origin by using the following transformations:

$$
\widetilde{\Phi_{t}}=x_{t}-\frac{d+\varepsilon}{c}, \widetilde{\Psi}_{t}=y_{t}-\frac{r^{*}+p-\varepsilon}{b} .
$$

From (47), (46) takes the following form:

$$
\begin{aligned}
\widetilde{\Phi_{t+1}}= & \left(1+h\left(r^{*}+p-\varepsilon\right)\right)\left(\widetilde{\Phi_{t}}+\frac{d+\varepsilon}{c}\right)-b h\left(\widetilde{\Phi_{t}}+\frac{d+\varepsilon}{c}\right)\left(\widetilde{\Psi}_{t}+\frac{r^{*}+p-\varepsilon}{b}\right)-\frac{d+\varepsilon}{c}, \widetilde{\Psi_{t+1}}=(1-h(d+\varepsilon))\left(\widetilde{\Psi}_{t}+\frac{r^{*}+p-\varepsilon}{b}\right) \\
& +\operatorname{ch}\left(\widetilde{\Phi_{t}}+\frac{d+\varepsilon}{c}\right)\left(\widetilde{\Psi}_{t}+\frac{r^{*}+p-\varepsilon}{b}\right)-\frac{r^{*}+p-\varepsilon}{b} .
\end{aligned}
$$

By Taylor series about $\left(\widetilde{\Phi_{t}}, \widetilde{\Psi_{t}}\right)=(0,0)$, one gets

$$
\widetilde{\Phi_{t+1}}=\widetilde{\Phi_{t}}-\frac{b h}{c}(d+\varepsilon) \widetilde{\Psi}_{t}-b h \widetilde{\Phi_{t}} \widetilde{\Psi}_{t}+h p \widetilde{\Phi_{t}}, \widetilde{\Psi_{t+1}}=\frac{c h}{b}(r-\varepsilon) \widetilde{\Phi_{t}}+\widetilde{\Psi}_{t}+c h \widetilde{\Phi_{t}} \widetilde{\Psi}_{t}
$$

Now by using following transformation 


$$
\left(\begin{array}{c}
\widetilde{\Phi_{t}} \\
\widetilde{\Psi}_{t}
\end{array}\right):=\left(\begin{array}{ccc}
-\frac{b}{c(r-\varepsilon)} \sqrt{(\varepsilon-r)(d+\varepsilon)} & \frac{b}{c(r-\varepsilon)} \sqrt{(\varepsilon-r)(d+\varepsilon)} \\
1 & 1
\end{array}\right)\left(\widetilde{X_{t}} \widetilde{Y}_{t}\right)
$$

linear part of (49) transforms into canonical form. In view of where (50), (49) becomes

$$
\left(\begin{array}{c}
\widetilde{X_{t+1}} \\
\widetilde{Y_{t+1}}
\end{array}\right)=\left(\begin{array}{cc}
-1 & 0 \\
0 & 3
\end{array}\right)+\left(\begin{array}{l}
\widetilde{F}\left(\widetilde{X}_{t}, \widetilde{Y}_{t}, p\right) \\
\widetilde{G}\left(\widetilde{X}_{t}, \widetilde{Y}_{t}, p\right)
\end{array}\right),
$$

$$
\widetilde{F}\left(\widetilde{X_{t}}, \widetilde{Y}_{t}, p\right)=-\frac{1}{2} b h{\widetilde{X_{t}}}^{2}+\frac{1}{2} b h \widetilde{Y}_{t}^{2}-\frac{1}{2} h p \widetilde{X_{t}}, \widetilde{G}\left(\widetilde{X_{t}}, \widetilde{Y}_{t}, p\right)=\frac{1}{2} \operatorname{chX_{t}^{2}}-\frac{1}{2} \operatorname{ch} \widetilde{Y}_{t}^{2}
$$

Now, for (51), center manifold $M^{c} E_{\text {trival }}$ at $E_{\text {trival }}$ is explored in a small nbhd of $p$. Therefore, $M^{c} E_{\text {trival }}$ can be expressed as follows:

$$
M^{c} E_{\text {trival }}=\left\{\left(\widetilde{X_{t}}, \widetilde{Y}_{t}\right): \widetilde{Y}_{t}=c_{0} p+c_{1}{\widetilde{X_{t}}}^{2}+c_{2} \widetilde{X_{t}} p+c_{3} p^{3}+O\left(\left(\left|\widetilde{X_{t}}\right|+|p|\right)^{3}\right)\right\} .
$$

After some manipulations, one gets

Finally, the map (51) restricting to $M^{c} E_{\text {trival }}$ is

$$
\begin{aligned}
& c_{0}=c_{2}=c_{3}=0, \\
& c_{1}=-\frac{1}{4} c h .
\end{aligned}
$$

$$
\widetilde{\mathrm{F}}\left(\widetilde{X_{t}}\right)=-\widetilde{X_{t}}+h_{1}{\widetilde{X_{t}}}^{2}+h_{2} \widetilde{X_{t}} p+h_{3}{\widetilde{X_{t}}}^{2} p+h_{4}{\widetilde{X_{t}}}^{2}+h_{5}{\widetilde{X_{t}}}^{3}+O\left(\left(\left|\widetilde{X_{t}}\right|+|p|\right)^{4}\right)
$$

with

$$
\begin{aligned}
& h_{1}=-\frac{1}{2} b h, \\
& h_{2}=-\frac{h}{2}, \\
& h_{3}=h_{4}=h_{5}=0 .
\end{aligned}
$$

In order for the map (51) to undergo flip bifurcation, the following discriminatory quantities are required to be nonzero $[28,29]$ :

$$
\begin{aligned}
& \hat{1}_{1}=\left.\left(\frac{\partial^{2} \widetilde{\mathrm{F}}}{\partial \widetilde{X_{t}} \partial p}+\frac{1}{2} \frac{\partial \widetilde{\mathrm{F}}}{\partial p} \frac{\partial^{2} \widetilde{\mathrm{F}}}{\partial{\widetilde{X_{t}}}^{2}}\right)\right|_{\left(\widetilde{X}_{t}, p\right)}=(0,0), \\
& \underset{2}{\wedge}=\left.\left(\frac{1}{6} \frac{\partial^{3} \widetilde{\mathrm{F}}}{\partial{\widetilde{X_{t}}}^{3}}+\left(\frac{1}{2} \frac{\partial^{2} \widetilde{\mathrm{F}}}{\partial \widetilde{X}_{t}^{2}}\right)^{2}\right)\right|_{\left(\tilde{X}_{t}, p\right)}=(0,0) .
\end{aligned}
$$

On computation, one gets $\wedge=-(h / 2) \neq 0$, but $\wedge=(1 / 6) b^{2} h^{2}>0$, which shows that model (4) undergoes flip bifurcation if $\left.(b, c, d, h, r, \varepsilon) \in F B\right|_{E_{\text {positive }}}$ and, in particular, stable period-2 points bifurcating from $E_{\text {positive }}$. 


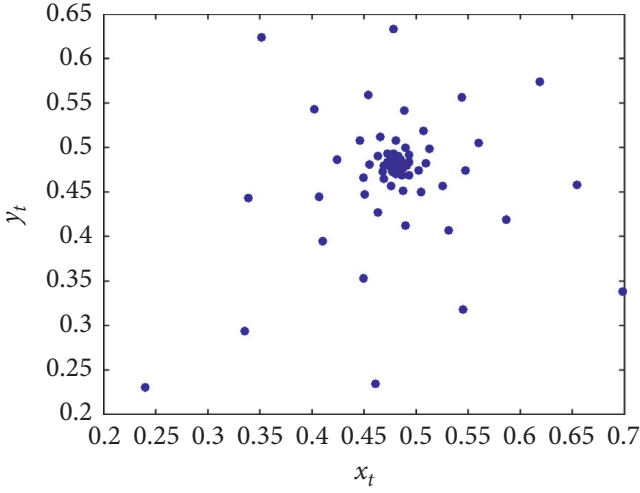

(a)

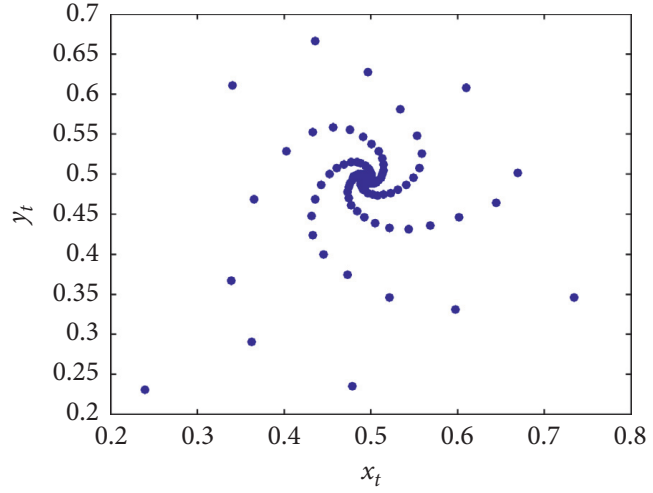

(b)

FIgURE 1: Stable node of model (4). (a) $r=2.1$ with $(0.24,0.23)$. (b) $r=2.3$ with $(0.4,0.5)$.

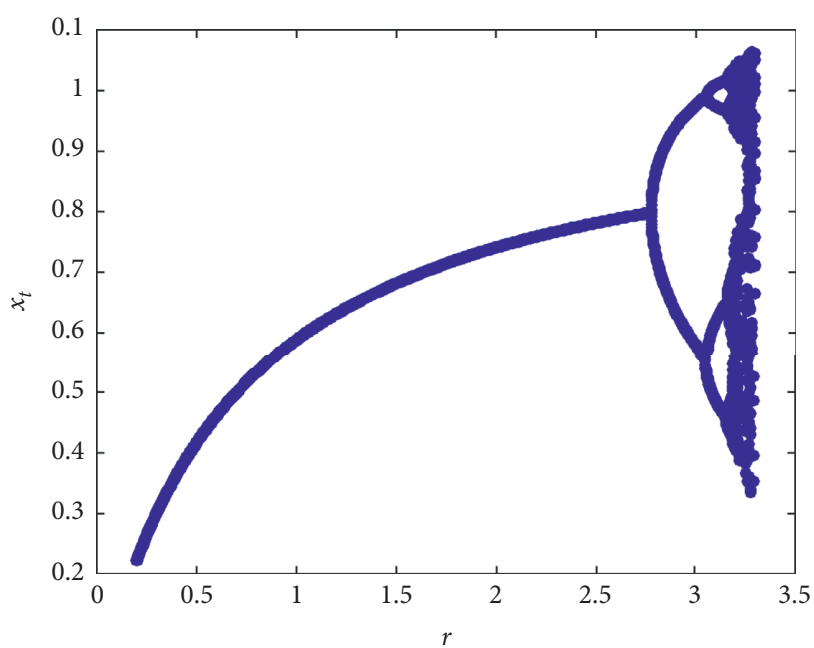

(a)

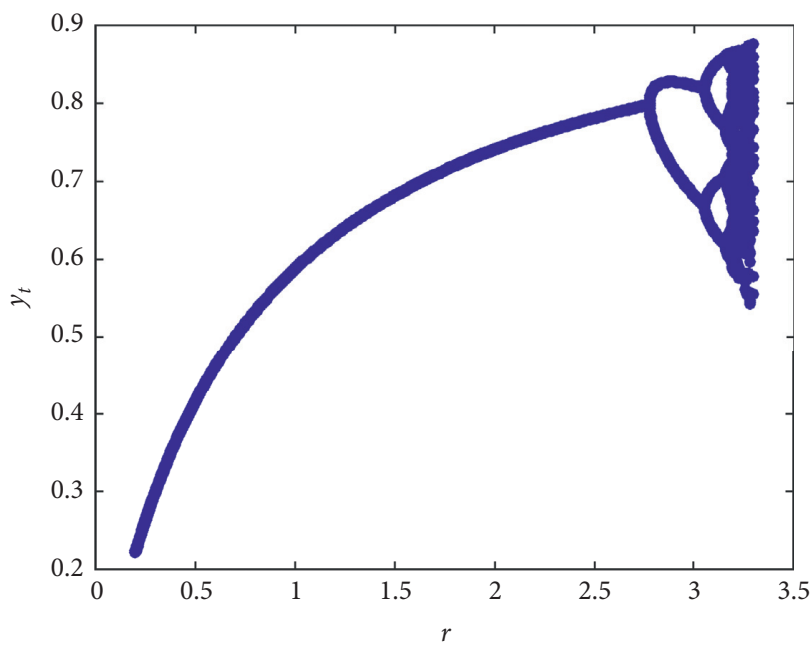

(b)

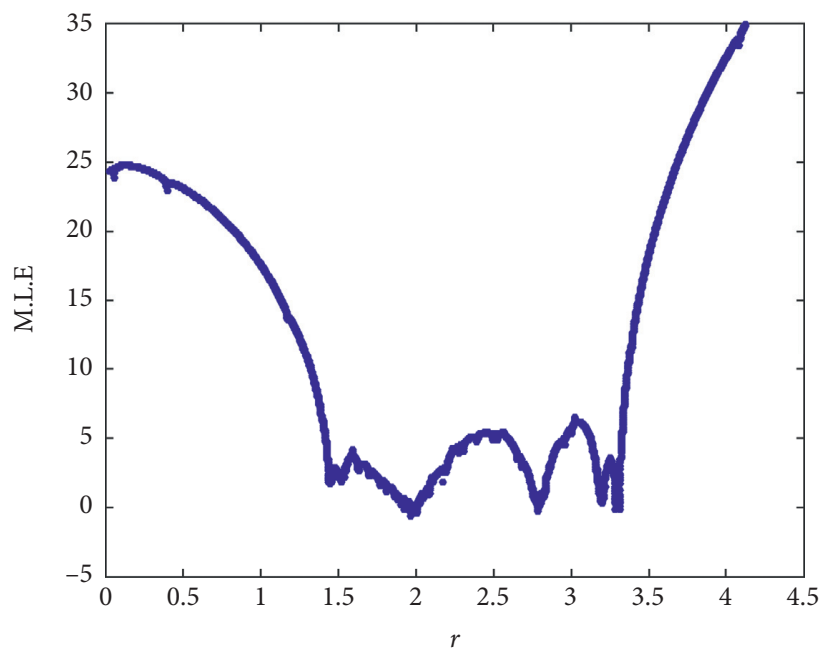

(c)

Figure 2: (a, b) Flip bifurcation diagram with $r \in[0.2,3.95]$ and $(0.24,0.25)$. (c) M.L.E corresponding to (a, b). 


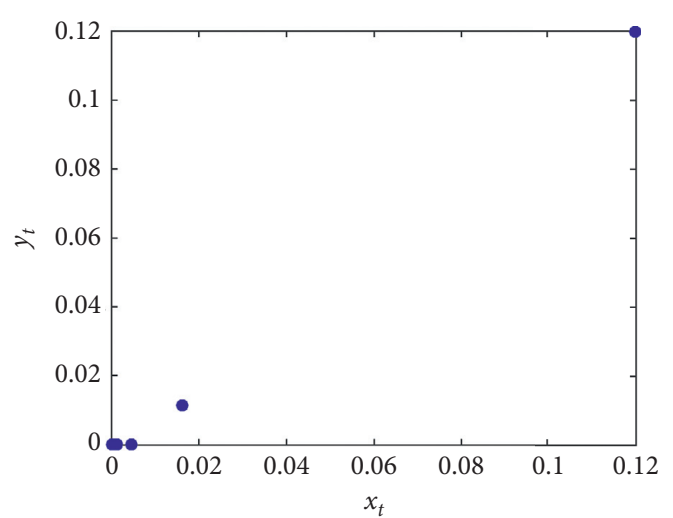

(a)

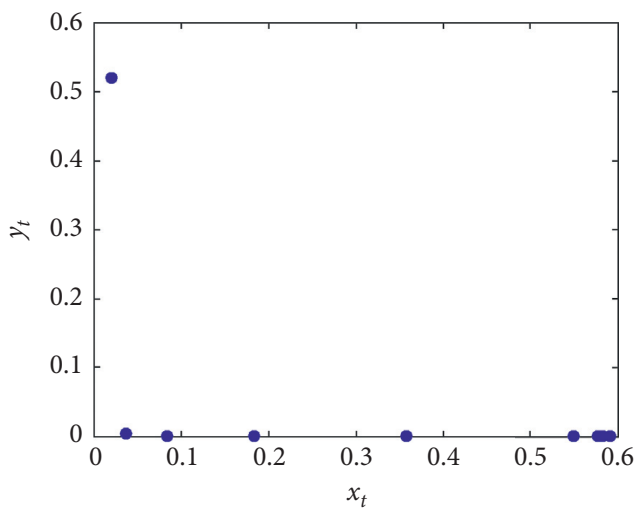

(c)

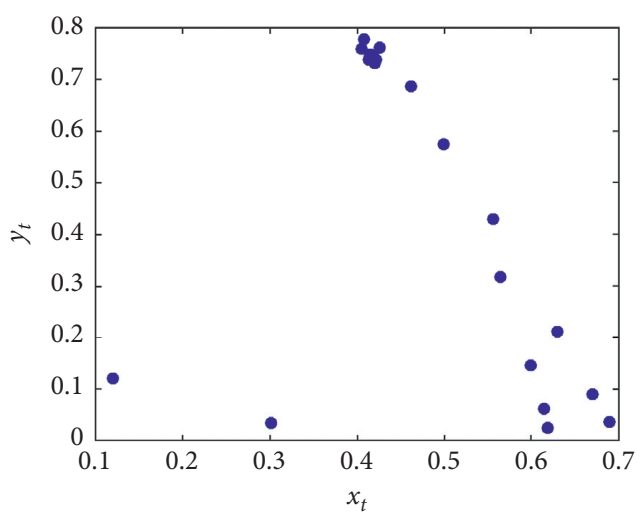

(e)

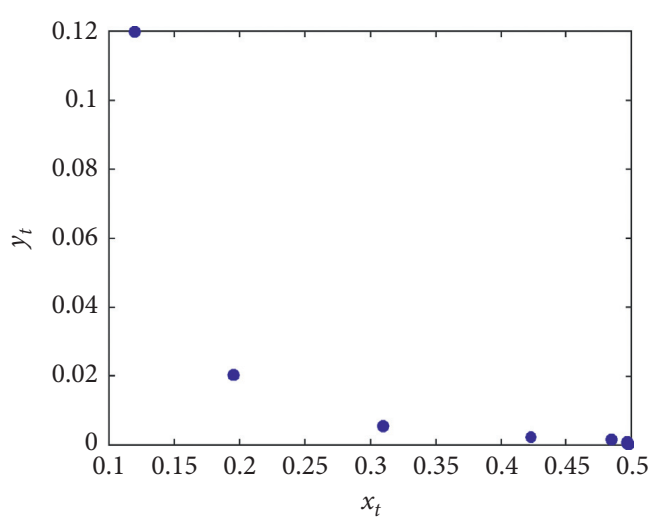

(b)

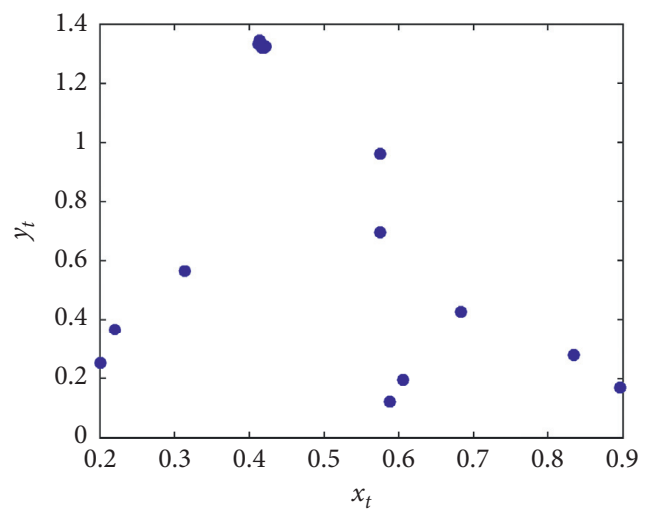

(d)

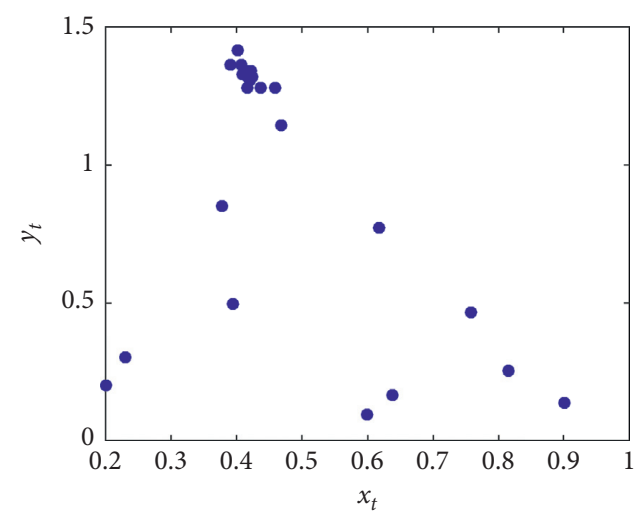

(f)

Figure 3: Complex dynamics of model (4). (a) $r=0.01$ with $(0.4,0.5)$. (b) $r=0.12$ with $(0.4,0.5)$. (c) $r=0.193$ with $(0.04,0.02)$. (d) $r=0.2112$ with $(0.61,0.61)$. (e) $r=0.21$ with $(0.0023,0.6)$. (f) $r=0.221$ with $(0.24,0.22)$.

\section{Numerical Simulations}

Here, some simulations will be presented in order to verify the obtained results in Sections 2 and 4. For instance, choose $\varepsilon=1.9, h=1$, then from (24), one gets $d>$ 0.2052631578947369. Furthermore, if $d=0.5>$ 0.2052631578947369 , then from (23), one gets $r>$ 0.23333333333333317 . But for the existence of unique positive fixed point, it is required that $r>\varepsilon=1$.9. So, for these numerical values and the condition on parameter $r$ where unique positive fixed point is stable focus is $r>\max \{0.23333333333333317,1.9\}$. Hence, if one choose parametric value $r=2.1$ which satisfies $r>\max$ $\{0.23333333333333317,1.9\}$ and $b=0.4, c=0.5$, then it is clear from Figure $1(\mathrm{a})$ that $E_{\text {positive }}=(4.8,0.5)$ is stable focus. Similarly, Figure 1(b) also shows that $E_{\text {positive }}$ of (4) is stable focus. Now, from (25), one can say that $E_{\text {positive }}$ of (4) is unstable focus if $r<0.23333333333333317$, and hence, flip bifurcation takes place if $r<0.23333333333333317$. For instance, if $r=0.09<0.23333333333333317$, then 


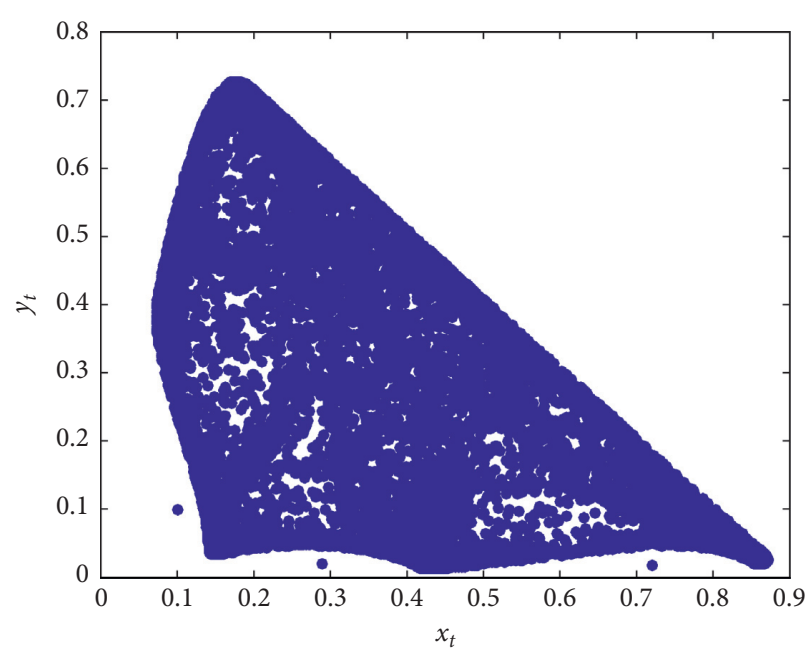

(a)

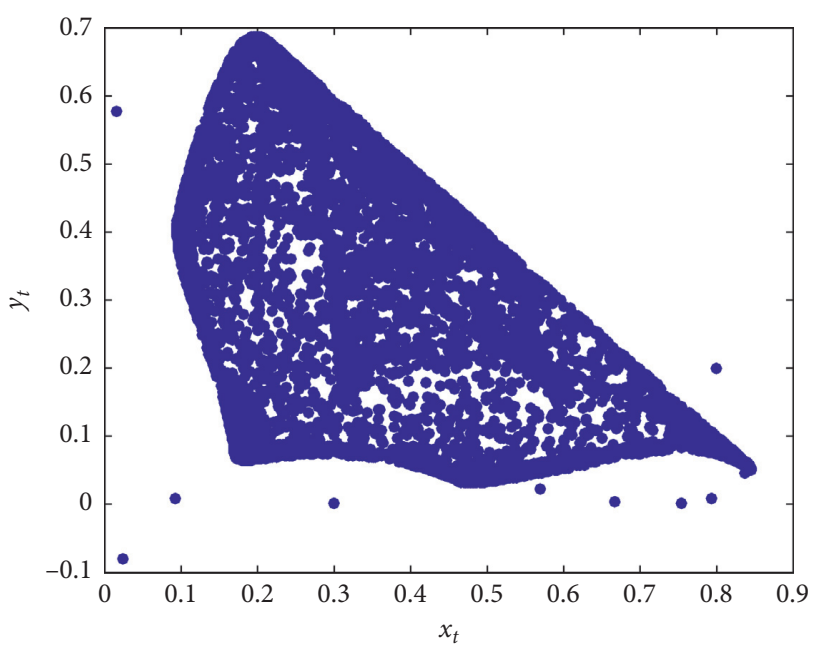

(b)

Figure 4: Strange attractors if $r=0.18$ (resp., $r=0.192$ ) with $(0.8,0.2)$.

$\zeta_{1}=-1.0842264752180841$,

but

$\zeta_{2}=3.084226475218084 \neq 1$ or -1 . Moreover, $\wedge=-0.5 \neq 0$ but $\wedge=0.026666666666666672>0$ which implies that stable period-2 points bifurcating from $E_{\text {positive }}$. Hence, this simulation agrees with theoretical results in Section 4. The $2 \mathrm{D}$ bifurcation diagrams with corresponding maximum Lyapunov exponents for said parametric values are plotted in Figure 2. Finally, the trajectories associated with Figures 2(a) and 2(b) are also plotted in Figures 3(a)-3(f) that indicates (4) exhibits complex dynamics having orbits of period-4, period-6, period-8, period-12, period-17, and period- 18 .

5.1. Fractal Dimension. It is defined by using Lyapunov exponents as follows [30, 31]:

$$
D_{L}=\mathrm{J}+\frac{\sum_{i=1}^{\mathrm{J}} \zeta_{\mathrm{J}}}{\left|\zeta_{\mathrm{J}}\right|},
$$

with $\zeta_{1}, \ldots, \zeta_{n}$ being Lyapunov exponents, where $\mathrm{J}$ is the largest integer s.t. $\sum_{i=1}^{\top} \zeta_{\mathrm{J}} \geq 0$ and $\sum_{i=1}^{J+1} \zeta_{\mathrm{J}}<0$. The fractal dimension for considered model (4) becomes

$$
D_{L}=1+\frac{\zeta_{1}}{\left|\zeta_{2}\right|} .
$$

Now for values of $d, \varepsilon, h, c, b$, and $r$, two Lyapunov exponents are numerically computed. If $d=0.5$, $\varepsilon=1.9, h=1, c=0.5, b=0.4$, then $\zeta_{1}=3.031748015872047$ (resp., $\left.\quad \zeta_{1}=3.024648117575002\right)$ and $\zeta_{2}=$ -1.0317480158720471 (resp., $\zeta_{2}=-1.0246481175750022$ for $r=0.18$ (resp., $r=0.192$ ). So fractal dimension for the model (4) is

$$
\begin{aligned}
d_{L} & =1+\frac{3.031748015872047}{|-1.0317480158720471|} \\
& =3.938457810659876 \text { for } r=0.18, \\
d_{L} & =1+\frac{3.024648117575002}{|-1.0246481175750022|} \\
& =3.951889595750518 \text { for } r=0.192 .
\end{aligned}
$$

For above chosen parametric values, strange attractors are also plotted in Figures 4(a) and 4(b) that demonstrate (4) has a complex dynamical behavior.

\section{Chaos Control}

By a state feedback control method, we will stabilize chaotic orbits at an unstable fixed point motivated from existing literature $[32,33]$. By adding control force $C_{t}$ to model $(4)$, then

$$
\begin{aligned}
x_{t+1} & =(1+h(r-\varepsilon)) x_{t}-b h x_{t} y_{t}+C_{t}, \\
y_{t+1} & =(1-h(d+\varepsilon)) y_{t}+c h x_{t} y_{t}, \\
C_{t} & =-g_{1}\left(x_{t}-\frac{d+\varepsilon}{c}\right)-g_{2}\left(y_{t}-\frac{r-\varepsilon}{b}\right),
\end{aligned}
$$

with $g_{1}$ and $g_{2}$ being feedback gains. Now, $\left.\Omega^{C}\right|_{E_{\text {positive }}}$ at $E_{\text {positive }}$ for the controlled system (61) is

$$
\left.\Omega^{C}\right|_{E_{\text {positive }}}:=\left(\begin{array}{cc}
1-g_{1} & -\frac{b h}{c}(d+\varepsilon)-g_{2} \\
\frac{c h}{b}(r-\varepsilon) & 1
\end{array}\right) \text {, }
$$




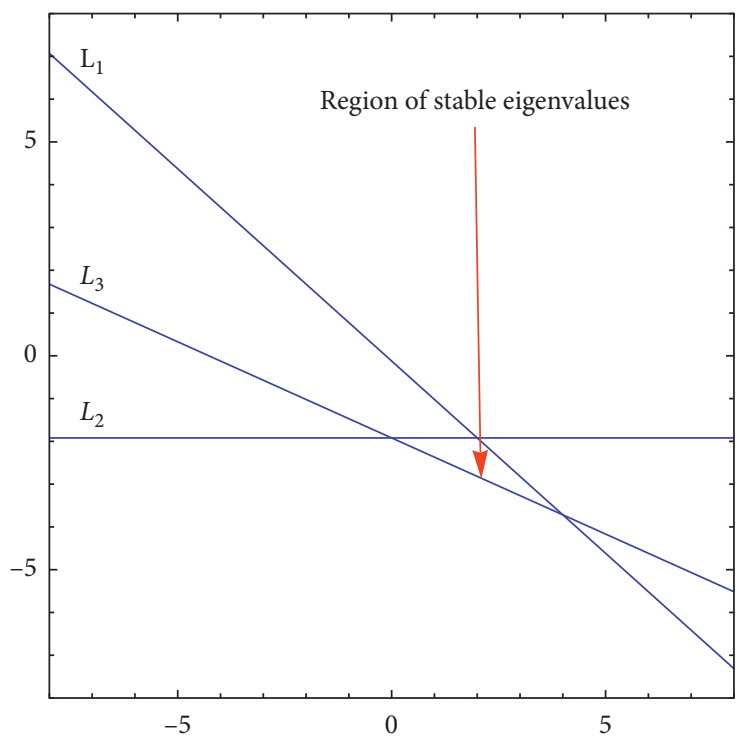

(a)

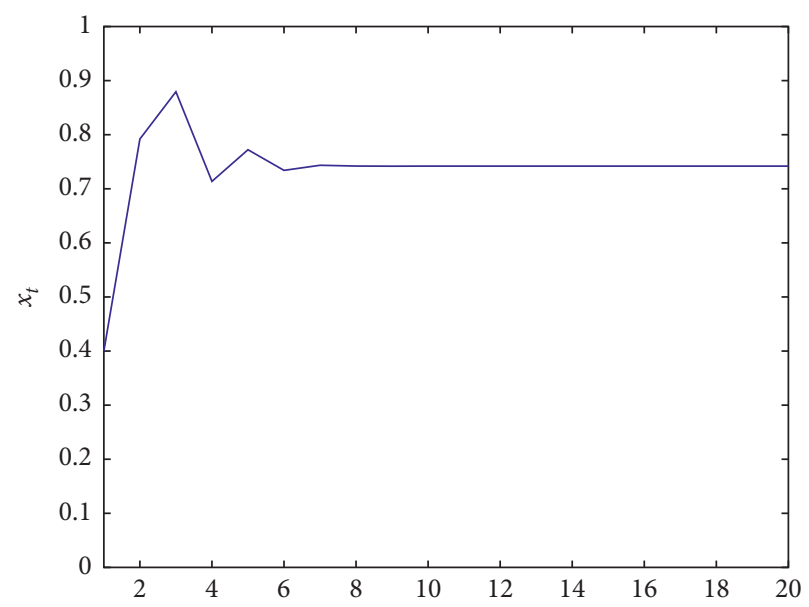

(b) $t$

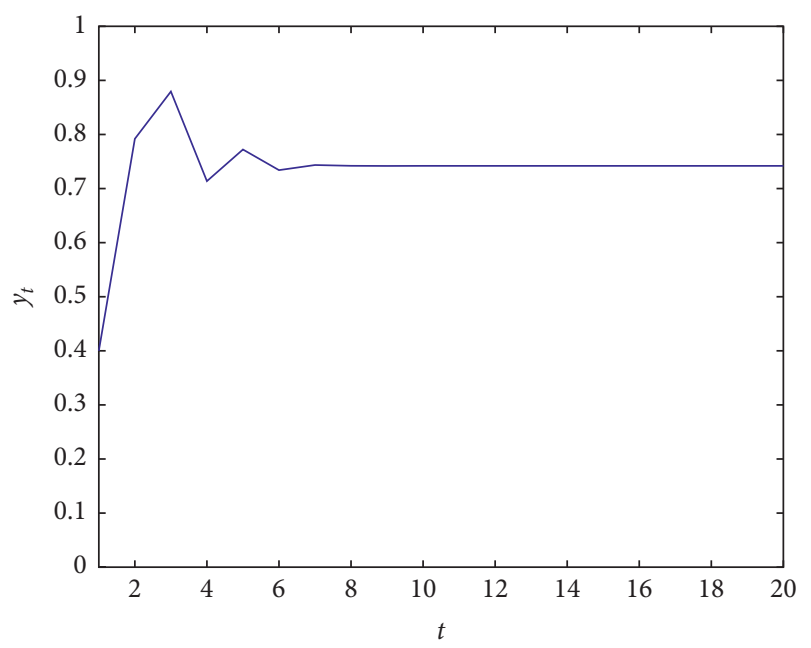

(c)

FiguRe 5: Control of chaotic trajectories of (61) for $d=0.5, \varepsilon=1.9, h=1, c=0.5, b=0.4$, and $r=0.12$ with (0.4, 0.5): (a) stability region in $\left(g_{1}, g_{2}\right)$-plane; $(\mathrm{b}, \mathrm{c})$ dynamics for $x_{t}$ and $y_{t}$, respectively.

whose auxiliary equation is

$$
\zeta_{C}^{2}-\mathscr{T}\left(E_{\text {positive }}\right) \zeta_{C}+\mathscr{D}\left(E_{\text {positive }}\right)=0
$$

with

$$
\begin{aligned}
& \mathscr{T}\left(E_{\text {positive }}\right)=2-g_{1}, \\
& \mathscr{D}\left(E_{\text {positive }}\right)=1-g_{1}+h^{2}(r-\varepsilon)(d+\varepsilon)+\frac{c h}{b}(r-\varepsilon) g_{2} .
\end{aligned}
$$

If $\zeta_{1,2}$ are roots of (63), then

$$
\begin{aligned}
\zeta_{1}+\zeta_{2} & =2-g_{1}, \\
\zeta_{1} \zeta_{2} & =1-g_{1}+h^{2}(r-\varepsilon)(d+\varepsilon)+\frac{c h}{b}(r-\varepsilon) g_{2} .
\end{aligned}
$$

Here, it is noted that solution of the following equations

$$
\begin{gathered}
\zeta_{1}=1, \\
\zeta_{1}=-1, \\
\zeta_{1} \zeta_{2}=1,
\end{gathered}
$$

gives the lines of marginal stability, which further grantee the fact that $\left|\zeta_{1,2}\right|<1$. From (66) and (69), one gets

$$
L_{1}:-g_{1}+\frac{c h}{b}(r-\varepsilon) g_{2}+h^{2}(r-\varepsilon)(d+\varepsilon)=0 .
$$

From (65), (66), and (67), one gets

$$
L_{2}: \frac{c h}{b}(r-\varepsilon) g_{2}+h^{2}(r-\varepsilon)(d+\varepsilon)=0 .
$$

From (65), (66), and (68), one gets 
TABle 2: Behavior of model (4) at $E_{\text {trival }}$ and $E_{\text {positive }}$

\begin{tabular}{lc}
\hline Fixed points & Respective behavior \\
\hline$E_{\text {trival }}$ & Sink if $0<h<\min \{(2 / \varepsilon-r) ;(2 / d+\varepsilon)\}$, where $\varepsilon>r$; source if $h>\max \{(2 / \varepsilon-r),(2 / d+\varepsilon)\}$, where $\varepsilon>r$ \\
& Saddle if $(2 / \varepsilon-r)<h<(2 / d+\varepsilon)$; nonhyperbolic if $h=(2 / \varepsilon-r)$ or $h=(2 / d+\varepsilon)$ \\
$E_{\text {positive }}$ & Never stable focus; unstable focus if $r>\varepsilon$; nonhyperbolic if $r=\varepsilon$ \\
& Stable node if $r>\left(\varepsilon h^{2}(d+\varepsilon)-4 / h^{2}(d+\varepsilon)\right) ;$ unstable node if $r<\left(\varepsilon h^{2}(d+\varepsilon)-4 / h^{2}(d+\varepsilon)\right)$ \\
Nonhyperbolic if $r=\left(\varepsilon h^{2}(d+\varepsilon)-4 / h^{2}(d+\varepsilon)\right)$
\end{tabular}

$$
L_{2}: 2 g_{1}-\frac{c h}{b}(r-\varepsilon) g_{2}-h^{2}(r-\varepsilon)(d+\varepsilon)=4
$$

Thus, $L_{1}, L_{2}$, and $L_{3}$ in $\left(g_{1}, g_{2}\right)$-plane determines the triangular region that gives $\left|\zeta_{1,2}\right|<1$ (see Figure 5(a)), whereas Figures 5(b) and 5(c) tell about $E_{\text {positive }}$ that the chaotic trajectories are stabilized. For the qualitative behavior of continuous dynamical systems, we refer the interested readers to [34-36] and references cited therein.

\section{Periodic Points of Model (4)}

Existence of periodic points at $E_{\text {trival }}$ and $E_{\text {positive }}$ is investigated in this section.

Theorem 3. $E_{\text {trival }}$ is a periodic point having prime period-1.

Proof. From (4), we have

$$
F(x, y):=(f(x, y), g(x, y)),
$$

where

$$
\begin{aligned}
& f(x, y)=(1+h(r-\varepsilon)) x-b h x y \\
& g(x, y)=(1-h(d+\varepsilon)) y+c h x y .
\end{aligned}
$$

From (73) along with (74), one gets the required results:

$$
\left.F\right|_{E_{\text {trival }}}=E_{\text {trival }} \text {. }
$$

Theorem 4. $E_{\text {positive }}$ is a periodic point having prime period1.

Proof. From (73) along with (74), one gets the required results:

$$
\left.F\right|_{E_{\text {positive }}}=E_{\text {positive }}
$$

Theorem 5. $E_{\text {trival }}$ is a periodic point of period-2,3,..,n.

Proof. From (73), one gets

$$
\begin{aligned}
F^{2}(x, y) & =((1+h(r-\varepsilon)) f(x, y)-b h f(x, y) g(x, y), \\
(1-h(d+\varepsilon)) g(x, y)+\operatorname{ch} f(x, y) g(x, y))\left.\Longrightarrow F^{2}\right|_{E_{\text {positive }}} & =E_{\text {positive }}, \\
F^{3}(x, y) & =\left((1+h(r-\varepsilon)) f^{2}(x, y)-b h f^{2}(x, y) g^{2}(x, y),\right. \\
\left.(1-h(d+\varepsilon)) g^{2}(x, y)+c h f^{2}(x, y) g^{2}(x, y)\right)\left.\Longrightarrow F^{3}\right|_{E_{\text {positive }}} & =E_{\text {positive }}, \\
\vdots & \\
F^{i}(x, y) & =\left((1+h(r-\varepsilon)) f^{i-1}(x, y)-b h f^{i-1}(x, y) g^{i-1}(x, y),\right. \\
\left.(1-h(d+\varepsilon)) g^{i-1}(x, y)+c h f^{i-1}(x, y) g^{i-1}(x, y)\right)\left.\Longrightarrow F^{i}\right|_{E_{\text {positive }}} & =E_{\text {positive }} .
\end{aligned}
$$

So, from (77), one gets the required statement.

Theorem 6. $E_{\text {positive }}$ is a periodic point of period $-2,3, \ldots, n$.

Proof. From (77), one gets the required statement:

$$
\begin{array}{r}
\left.F^{2}\right|_{E_{\text {positive }}}=E_{\text {positive }}, \\
\left.F^{3}\right|_{E_{\text {positive }}}=E_{\text {positive }}, \\
\vdots \\
\left.F^{i}\right|_{E_{\text {positive }}}=E_{\text {positive }}
\end{array}
$$

\section{Conclusion}

In this paper, we have investigated the topological classifications at fixed points, bifurcation analysis, and chaos in a model, which is depicted in (4). It is examined that $\forall d, \varepsilon, h, c, b, r$ model has a trivial fixed point: $E_{\text {trival }}$ and the unique positive fixed point: $E_{\text {positive }}$ if $r>\varepsilon$. By existing linear theory of stability, we have studied the topological classifications at $E_{\text {trival }}$ and $E_{\text {positive }}$, and conclusion is reported in Table 2. Furthermore, we have examined the existence of possible bifurcations at $E_{\text {trival }}$ and $E_{\text {positive }}$ and proved that model (4) does not undergo flip bifurcation if $\left.(b, c, d, h, r, \varepsilon) \in \mathrm{FB}\right|_{E_{\text {trival }}}$, and hence, $E_{\text {trival }}$ is degenerated 
with a higher codimension. We have also examined that if $\left.(b, c, d, h, r, \varepsilon) \in \mathrm{HB}\right|_{E_{\text {positive }}}$, then at $E_{\text {positive }}$, model (4) does not undergo N-S bifurcation. Moreover, at fixed point $E_{\text {positive }}$, we have proved that model (4) undergoes the flip bifurcation if $\left.(b, c, d, h, r, \varepsilon) \in \mathrm{FB}\right|_{E_{\text {positive }}}$. Some numerical simulations are performed not only to demonstrate obtained theoretical results but also to tell the complex behaviors in orbits of period-4, period-6, period-8, period-12, period-17, and period-18. We have also computed maximum Lyapunov exponents numerically. By the feedback control method, we have stabilized chaos existing in the considered model. Finally, existence of periodic points at $E_{\text {trival }}$ and $E_{\text {positive }}$ of model (4) is explored.

\section{Data Availability}

All the data utilized in this article have been included, and the sources adopted are cited accordingly.

\section{Conflicts of Interest}

The authors declare that they have no conflicts of interest regarding the publication of this paper.

\section{Acknowledgments}

This project was funded by the Deanship of Scientific Research (DSR) at King Abdulaziz University, Jeddah, under Grant no. G: 589-130-1441. The authors, therefore, acknowledge with thanks DSR for technical and financial support.

\section{References}

[1] M. Braun, Differential Equations and Their Applications: An Introduction to Applied Mathematics, Springer-Verlage New York, New York, NY, USA, 1983.

[2] L. J. S. Allen, Introduction to Mathematical Biology, Pearson/ Prentice Hall, Upper Saddle River, NJ, USA, 2007.

[3] F. Brauer, C. Castillo-Chavez, and C. Castillo-Chavez, Mathematical Models in Population Biology and Epidemiology, Springer, New York, NY, USA, 2001.

[4] T. Räz, "The volterra principle generalized," Philosophy of Science, vol. 84, no. 4, pp. 737-760, 2017.

[5] A. Q. Khan and T. Khalique, "Bifurcations and chaos control in a discrete-time biological model," International Journal of Biomathematics, vol. 13, pp. 1-35, 2020.

[6] J. R. Beddington, C. A. Free, and J. H. Lawton, "Dynamic complexity in predator-prey models framed in difference equations," Nature, vol. 255, no. 5503, pp. 58-60, 1975.

[7] F. Chen, "Permanence and global attractivity of a discrete multispecies Lotka-Volterra competition predator-prey systems," Applied Mathematics and Computation, vol. 182, no. 1, pp. 3-12, 2006.

[8] X. Chen, "Periodicity in a nonlinear discrete predator-prey system with state dependent delays," Nonlinear Analysis: Real World Applications, vol. 8, no. 2, pp. 435-446, 2007.

[9] N. Fang and X. X. Chen, "Permanence of a discrete multispecies lotka-volterra competition predator-prey system with delays," Nonlinear Analysis: Real World Applications, vol. 9, no. 5, pp. 2185-2195, 2008.
[10] Q. Fang, X. Li, and M. Cao, "Dynamics of a discrete predatorprey system with beddington-deangelis function response," Applied Mathematics, vol. 03, no. 04, pp. 389-394, 2012.

[11] E.-G. Gu, "The nonlinear analysis on a discrete host-parasitoid model with pesticidal interference," Communications in Nonlinear Science and Numerical Simulation, vol. 14, no. 6, pp. 2720-2727, 2009.

[12] H. N. Agiza, E. M. ELabbasy, H. EL-Metwally, and A. A. Elsadany, "Chaotic dynamics of a discrete prey-predator model with holling type ii," Nonlinear Analysis: Real World Applications, vol. 10, no. 1, pp. 116-129, 2009.

[13] H.-F. Huo and W.-T. Li, "Stable periodic solution of the discrete periodic leslie-gower predator-prey model," Mathematical and Computer Modelling, vol. 40, no. 3-4, pp. 261-269, 2004.

[14] L. Li and J. Zhi, "Global stability of periodic solutions for a discrete predator-prey system with functional response," Nonlinear Dynamics, vol. 72, pp. 507-516, 2003.

[15] C. Lu and L. Zhang, "Permanence and global attractivity of a discrete semi-ratio dependent predator-prey system with holling ii type functional response," Journal of Applied Mathematics and Computing, vol. 33, no. 1-2, pp. 125-135, 2010.

[16] X. Liu and D. Xiao, "Complex dynamic behaviors of a discrete-time predator-prey system," Chaos, Solitons \& Fractals, vol. 32, no. 1, pp. 80-94, 2007.

[17] M. T. Morgan, W. G. Wilson, and T. M. Knight, "Plant population dynamics, pollinator foraging, and the selection of self-fertilization," The American Naturalist, vol. 166, no. 2, pp. 169-183, 2005.

[18] M. Zhao and L. Zhang, "Permanence and chaos in a hostparasitoid model with prolonged diapause for the host," Communications in Nonlinear Science and Numerical Simulation, vol. 14, no. 12, pp. 4197-4203, 2009.

[19] M. Zhao, L. Zhang, and J. Zhu, "Dynamics of a host-parasitoid model with prolonged diapause for parasitoid," Communications in Nonlinear Science and Numerical Simulation, vol. 16, no. 1, pp. 455-462, 2011.

[20] L. Zhu and M. Zhao, "Dynamic complexity of a host-parasitoid ecological model with the Hassell growth function for the host," Chaos, Solitons \& Fractals, vol. 39, no. 3, pp. 1259-1269, 2009.

[21] M. R. S. Kulenović and G. Ladas, Dynamics of Second-Order Rational Difference Equations, Chapman and Hall/CRC, London, 2002.

[22] Z. Hu, Z. Teng, and L. Zhang, "Stability and bifurcation analysis of a discrete predator-prey model with nonmonotonic functional response," Nonlinear Analysis: Real World Applications, vol. 12, no. 4, pp. 2356-2377, 2011.

[23] A. Q. Khan, J. Ma, and D. Xiao, "Bifurcations of a two-dimensional discrete time plant-herbivore system," Communications in Nonlinear Science and Numerical Simulation, vol. 39, no. 2016, pp. 185-198, 2016.

[24] A. Q. Khan, J. Ma, and D. Xiao, "Global dynamics and bifurcation analysis of a host-parasitoid model with strong Allee effect," Journal of Biological Dynamics, vol. 11, no. 1, pp. 121-146, 2017.

[25] Z. Jing and J. Yang, "Bifurcation and chaos in discrete-time predator-prey system," Chaos, Solitons \& Fractals, vol. 27, no. 1, pp. 259-277, 2006.

[26] C.-H. Zhang, X.-P. Yan, and G.-H. Cui, "Hopf bifurcations in a predator-prey system with a discrete delay and a distributed delay," Nonlinear Analysis: Real World Applications, vol. 11, no. 5, pp. 4141-4153, 2010.

[27] M. Sen, M. Banerjee, and A. Morozov, "Bifurcation analysis of a ratio-dependent prey-predator model with the Allee effect," Ecological Complexity, vol. 11, pp. 12-27, 2012. 
[28] J. Guckenheimer and P. Holmes, Nonlinear Oscillations, Dynamical Systems and Bifurcation of Vector Fields, SpringerVerlag, New York, NY, USA, 1983.

[29] Y. A. Kuznetsov, Elements of Applied Bifurcation Theorey, Springer-Verlag New York, New York, NY, USA, 3rd edition, 2004.

[30] J. H. E. Cartwright, "Nonlinear stiffness, Lyapunov exponents, and attractor dimension," Physics Letters A, vol. 264, no. 4, pp. 298-302, 1999.

[31] J. L. Kaplan and J. A. Yorke, "Preturbulence: a regime observed in a fluid flow model of Lorenz," Communications in Mathematical Physics, vol. 67, no. 2, pp. 93-108, 1979.

[32] S. N. Elaydi, An Introduction to Difference Equations, Springer-Verlag, New York, NY, USA, 1996.

[33] S. Lynch, Dynamical Systems with Applications Using Mathematica, Birkhäuser, Boston, MA, USA, 2007.

[34] C. Tunç, "A note on boundedness of solutions to a class of non-autonomous differential equations of second order," Applicable Analysis and Discrete Mathematics, vol. 4, no. 2, pp. 361-372, 2010.

[35] C. Tunç, "Stability and boundedness of solutions of nonautonomous differential equations of second-order," Journal of Computational Analysis and Applications, vol. 13, no. 6, pp. 1067-1074, 2011.

[36] C. Tunç and O. Tunç, "A note on certain qualitative properties of a second order linear differential system," Applied Mathematics \& Information Sciences, vol. 9, no. 2, pp. 953-956, 2015. 